Available online on 15.07.2018 at http://jddtonline.info
Journal Of Drug Delivery and Therapeutics
Open Access to Pharmaceutical and Medical Research
(2011-18, publisher and licensee JDDT, This is an Open Access article which permits unrestricted non-
commercial use, provided the original work is properly cited

Open $\odot$ Access

Research Article

\title{
LIQUID CRYSTALLINE DRUG DELIVERY SYSTEM FOR SUSTAINED RELEASE LOADED WITH AN ANTITUBERCULAR DRUG
}

\author{
Ola Monika, Bhaskar Rajveer *, Patil Gaurav R* \\ Department of Pharmaceutics \& Quality assurance, R. C. Patel Institute of Pharmaceutical Education and Research, Near Karvand \\ Naka, Shirpur Dhule (M.S) India. 425405
}

\begin{abstract}
In present study, LCs were formulated and evaluate for desirable properties, Sonication conditions were firstly investigated to determine their effects on the morphological and dimensional characteristics of liquid crystals and optimized according probe sonication condition (Ultra Tarrux T25), liquid crystals with reproducible narrow particle size distribution and mean particle size of $168.0 \pm 2.1 \mathrm{~nm}$ were obtained. The structure of the dispersed cubosomes was revealed by XRD (X-ray diffraction) and SEM (scanning electron microscopy) as a liquid crystalline phase. To overcome the dose frequency and increase drug loading rate, in vitro-dissolution, method, ultracentrifuge be firstly develop liquid crystals containing Rifampicin. The encapsulation efficiency determined by UV spectroscopy was $93.86 \pm 0.11 \%$ and stability studies in $\mathrm{pH} 6.8$ phosphate buffer solutions further confirmed that Rifampicin was successfully encapsulated in liquid crystals.
\end{abstract}

Keywords: Cubic phases, liquid crystals, GMO, Rifampicin, high speed homogenization (Ultra Tarrux T25), probe sonication.

Article Info: Received 10 Feb, 2018; Review Completed 21 May 2018; Accepted 25 May 2018; Available online 15 July 2018

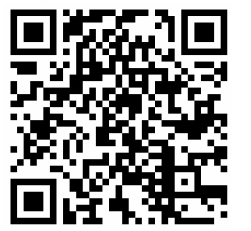

Cite this article as:

Ola M, Bhaskar R, Patil GR, Liquid crystalline drug delivery system for sustained release loaded with an antitubercular drug, Journal of Drug Delivery and Therapeutics. 2018; 8(4):93-101 DOI: http://dx.doi.org/10.22270/jddt.v8i4.1719

Department of Pharmaceutics \& Quality Assurance, R. C. Patel Institute of Pharmaceutical Education and Research, Near Karvand Naka, Shirpur, (M.S) India.

\section{INTRODUCTION}

Tuberculosis (TB) is a extremely catching and deadly respiratory system disease ${ }^{1}$ that affects millions of people around the world. TB treatment can take anywhere from six months to years to be effective. This length of moment in time can lead to different problems, including drug resistance, poor patient compliance, drug interactions and side effects. Improper use of first-line TB drugs contributes to multidrug-resistant $\mathrm{TB}^{2-3}$ .Although second-line drugs are available to treat MDR$\mathrm{TB}$, they are more toxic, expensive and less effective than first-line drugs. Therefore, 4it's important to improve the rational and optimal use of first-line drugs to avoid development of rapidly emerging MDR-TB. Decreasing the duration of treatment and dosing frequency may help to avoid drug resistance to first-line drugs, and reduce associated treatment costs and side effects, and improve compliance. A drug carrier system that protects first-line drugs from premature degradation, promotes sustained release and delivers the drug at targeted tissue may help to improve the efficacy of TB treatment ${ }^{4}$. Targeted delivery of drug molecules to organs or special sites is one of the most challenging research areas in pharmaceutical sciences. In colloidal delivery systems such as liposomes, micelles and nanoparticles a new frontier was opened for improving drug delivery. Nanoparticles can be administered via different routes of administration such as parenteral, oral, intraocular, transdermal or pulmonary inhalation. The German group produced the SLN by high pressure homogenization, while Prof. Gasco used a microemulsion technique. Solid lipid nanoparticles (SLNs) are produced by replacing the oil of an o/w emulsion by a solid lipid or a blend of solid lipids, i.e. the lipid particle matrix being solid at both room and body temperatures but most of problem occurred over their such as unpredictable 
gelation tendency, high water content dispersion and particle growth ${ }^{5}$. Nanotechnology is now being broadly of applied science and technology, for manipulating the structure of matter on molecular level at an incredibly small scale between 1-100nm.

The liquid crystalline state combines properties of both liquid and solid states. The liquid state is associated with the ability to flow, whereas the solid state is characterized by an ordered, crystalline structure. Crystalline solids exhibit short as well as long-range order with regard to both position and orientation of the molecules. Liquids are amorphous in general but may show short-range order with respect to position and/or orientation ${ }^{6}$. Crystalline solid characterized by longrange positional and orientational order in three dimensions. Self-assemble amphiphilic molecules (i.e., molecules with hydrophobic and hydrophilic character) including some lipids in aqueous system is known to form a variety of liquid crystalline phases such as lamellar, inverted hexagonal, and inverted cubic phases ${ }^{7}$. Lipid have been widely used as main constituent in various drug delivery systems, such as liposome, solid lipid nanoparticles, nanostructure lipid carriers, and lipid based liquid crystals. Among them, lipid-based liquid crystals highly ordered, thermodynamically stable internal nanostructure, thereby offering the potential as sustained drug release matrix ${ }^{8}$.

\section{Classification of Liquid Crystals ${ }^{9}$}

LCs are differentiated on the basis of positional order (i.e. molecule are arranged in randomly structure lattice) and orientational order (i.e. molecule are mostly pointed in the same direction). Moreover order can be either short-range (only between the molecule to each other) or long-range (extending to larger, sometimes macroscopic). LCs mainly classified as Lyotropic (LLCs) and Thermotropic (TLCs), physicochemical parameters responsible for the phase transitions $9-10$ classification of liquid crystals are as following:

1. Lyotropic liquid crystals,

a) Lamellar LCs

b) Hexagonal LCs

c) Cubic LCs

2. Thermotropic liquid crystals

a) Smectic liquid crystal

b) Nematic liquid crystal

c) Cholesteric liquid crystals

d) Discotic liquid crystals

Method of preparation of LCs. ${ }^{11}$

\section{(a) Probe Sonication}

High shear homogenization and ultrasound are dispersing techniques which were initially used for the production of solid lipid nanodispersion. However, its quality is compromised by the presence of microparticles. A pre-emulsion was obtained under stirring with an Ultra-Turrax T25 by adding melted lipid to a mixture of surfactants and water. A sonication probe was placed in this pre-emulsion which lead to droplet breakage by acoustic cavitationsand subsequent formation of oil in water $(\mathrm{o} / \mathrm{w})$ nanoemulsion which immediately cooled down to room temperature to generate liquid crystals ${ }^{5}$.

Advantages

1. Both methods are widespread and easy to handle

2. Equipments whatever use here are very common in every lab

3. Reduced shear stress

Disadvantages

1. Potential metal contamination

2. Physical instability like particle growth upon storage

\section{(b) Spray drying}

To widen the applications of cubosomes in pharmaceutical field, dry powder precursors can be fabricated by spray drying and used for the preparation of oral solid formulations and inhalants. This approach was originally proposed and investigated by scientist Spicer. In his research, the powder precursor could be prepared through drying a pre-dispersed aqueous solution that consisted of GMO, hydrophobically modified starch and water or contained GMO, dextran, ethanol and water, and then the colloidally stable dispersions of nano-structured cubosomes could be created by hydration of the precursors. Afterward $\mathbf{1 2}$ prepared GMO based cubosomes precursor containing diclofenac sodium through spray drying. The precursor was proven to have more effective and prolonged antiinflammatory and analgesic activity than pure drug when administered per orally; it is noteworthy, however, that residual solvent content is still a problem that cannot be ignored.

\section{Advantages:}

1) Spray drying technique is useful for powder formulation such as DPI (Dry powder inhaler, dry syrup).

2) This technique used for microencapsulation.

3) Organic solvent can use in this method.

Disadvantages:

1) From this method has low yield of formulation as 5$30 \%$ out of $100 \%$.

2) Spray drying method is complicated as compare to other method

\section{(c) Bottom-up approach}

Compared with the top-down approach, this dilutionbased approach can produce cubosomes without laborious fragmentation. In other words, it needs less energy input. Moreover, this approach is far more efficient at generating small particles. The reason for this might relate to the forming mechanism of cubosomes. The dilution-based approach can be regarded as a process of small particles forming big particles through aggregation, which is analogous to the use of precipitation processes to produce nanoparticles, whereas the top-down approach is more analogous to the attrition of big particles. In addition, cubosomes 
prepared through dilution show long term stability, which might be attributed to the homodisperse stabilizers onto the surface of cubosomes ${ }^{\mathbf{1 3}}$. Indeed, the use of hydrotrope can simplify the preparation process and produce cubosomes possessing similar or even better properties than those fabricated by the top-down approach. It should be noted, however, that this process via dilution is a pathway by charting trajectories on the ternary phase diagram (lipid and water hydrotrope), which requires knowledge of the full phase behavior; hence, the extent of dilution is difficult to control precisely. Owing to the addition of hydrotrope, many issues arise, such as the effects exerted by varying concentrations of hydrotrope on the physicochemical properties of LLC nanoparticles and the possible occurrence of irritation and allergic response when the mesophase formulations are administered. Finally, this bottom- up approach cannot effectively avoid forming vesicles.

\section{Advantages:}

1) Lower energy input.

2) Less time consuming process.

3) At high concentration prevent the formation of LCs.

4) No need the organic solvent

\section{Disadvantages:}

1) Milky white formulation formed.

2) Hydrotrope which shows allergic reaction when the mesophase formulation administered orally.

\section{(d) Heat treatment}

The coexistence of cubosomes with vesicles is speculated to provide multiphase manipulation of the sustained release of drugs; hence, to better investigate the release behavior of plain mesophases, vesicles should be eliminated as much as possible. In this case, heat treatment can be regarded as a good approach. Note that in the strictest sense, heat treatment is not an integrated process for the manufacture of cubosomes because it only promotes the transformation from noncubic vesicles to well-ordered cubic particles. The dispersed particles, therefore, can be produced by a simple processing scheme comprising a homogenization and heat-treatment step. From the reported studies, heat treatment could cause a decrease in the small particle size fraction that corresponded to vesicles and form more cubic phases with narrow particle distribution and good colloidal stability ${ }^{\mathbf{1 4}}$. Taking the whole process of preparation into account, it is obvious that the transition takes place during the procedure of heat treatment. The reason for transition could be speculated as an elevated temperature giving rise to a reduction in solubility and stability. When the temperature was below cloud point, the surfactant had a high solubility and thus the particles could exist stably and the phenomenon of fusion was hardly observed. Once reaching cloud point, the solubility of surfactant decreased notably and a notable fast fusion among vesicles would occur. Although masses of vesicles can transform to cubic nanoparticles through heat treatment, it does not mean that all the LLC systems are suitable for this procedure in particular, the systems loading drugs that cannot provide sufficient stability under the condition of high temperature (usually above $120^{\circ} \mathrm{C}$ ), such as some proteins and temperaturesensitive drugs are not suitable.

\section{Advantages:}

1) It produced good colloidal dispersion.

2) It can reduce particle size.

Disadvantages:

1) Degradation of thermo sensitive substance due to formation of aggregate.

Reduction of stability of formulation

\section{(e) Top-down approach}

The extreme viscous bulk phase is prepared by mixing structure-forming lipids with stabilizers, and then the resultant is dispersed into aqueous solution through the input of high energy such as high-pressure homogenization (HPH), sonication or shearing to form LLC nanoparticles. At present, HPH is the most extensively used technique in the preparation of LLC nanoparticles 13. Cubosomes. Based on the results observed, the concentration of F127 and temperature during HPH were regarded as crucially important parameters. Recently, a novel approach of shearing was proposed to fabricate LLC nanoparticles using a laboratory built- Shearing apparatus. Compared with the well-established ultrasonication approach, the shearing treatment could effectively prepare more stable and homogeneous cubosomes or hexosomes with high content of the hydrophobic phase (oil + lipophilic additives) within a short time (less than one minute). It seems that the preparation procedure is simple enough to be realized conveniently. In fact, the operation units in this procedure require several cycles to achieve the desired Nanoparticles with appropriate characteristics and the high-energy input is also regarded as a barrier to the temperature resensitive ingredients ${ }^{\mathbf{1 4}}$.

\section{Advantages:}

1) Lower impact to overall organization.

2) Visibility of formulation changes is clear.

3) No need of organic solvent.

4) Simple method as compare to other method such as spray drying.

\section{Disadvantages:}

1) Solution provides limited coverage in the first phase.

2) High energy input required.

3) Time consuming process.

\section{Applications of liquid crystals system: ${ }^{15}$}

Therapeutic compounds of diverse physicochemical properties such as analgesic, antibiotics, antifungal, anticancer, vitamins, antiasthamatics, immunosuppressive etc. monoglyceride based cubosome dispersion can be proposed for topical used, such as for precutaneous or mucosal applications. Because of the microbicidal properties of monoglycerides, could be used to design intravaginal treatment of sexually transmitted diseases caused by viruses (e.g. HSV, HIV) 
or by bacteria (e.g. Chlamydia trachomatis and neisseriagenorrticae). The cubosome technology is used to develop a synthetic venix the chessy white substance that coats infants in late gestation to help premature infants who are born without it. E vernix is a complex mixture of lipid (fat), proteins and water. Cubosome can also be used for controlled release application. Cubosome particles are used as oil water emulsion stabilizers and pollutant absorbants in cosmetics. More recent use is about personal cure product areas as varied as skin care, hair care, cosmetics and antiperspirant.

\section{MATERIALS AND METHODS}

The model Rifampicin was provided from Healthy Life Pharma Pvt. Ltd. Mumbai. Glycerol Monooleate was purchased from Abitec Corporation 1530 Jackson St Janesville, WI 53546 P- F68 (Poloxamer 188) was purchased from HI Media Lab. Pvt. Ltd. Mumbai. And ethanol from MerckSpecialities Pvt. Ltd., Worli, Mumbai.

\section{Methods}

\section{Drug-Excipient Interaction Study}

Fourier transformed infrared spectroscopy:

IR spectroscopy was used to determine the molecular interaction between Excipient and drug. The all physical mixtures and drug sample were mixed with dried $\mathrm{KBr}$ in ratio 1:100. Then small fraction of mixture was compressed on automatic IR press (KimayaEngg. Thane, India) pressure 10 tones to form transparent pellet. Then the IR spectrum of pellet was taken on FTIR spectrophotometer.

Method of Preparation of Liquid Crystals (LCs): ${ }^{7-11}$

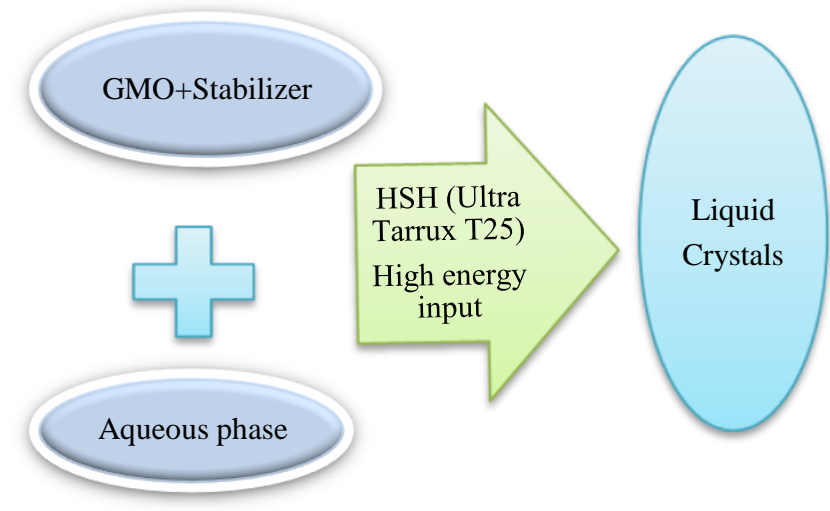

Figure 1: Preparation of liquid crystals

\section{Formulation of liquid crystals by Probe Sonication method (Ultra-Turrax T25)}

probe sonication method a pre-emulsion was obtained under stirring with an Ultra-Turrax T25 by adding melted lipid to a mixture of surfactants and water. A sonication probe was placed in this pre-emulsion which lead to droplet breakage by acoustic cavitationsand subsequent formation of oil in water $(\mathrm{o} / \mathrm{w})$ nanoemulsion which immediately cooled down to room temperature to generate Rifampicin-LCs. we obtain the expected particle size and PDI by using above technique. ${ }^{7-11}$

Table 1: Composition of formulation batches

\begin{tabular}{|l|l|l|l|l|l|l|}
\hline Sr.No & Batch no & Drug (mg) & GMO (\%) & Poloxamer $(\%)$ & Ethanol (\%) & Water (\%) \\
\hline 1 & B1 & 100 & 5.5 & 0.5 & 2 & 2 \\
\hline 2 & B2 & 100 & 5.5 & 1.0 & 2 & 2 \\
\hline 3 & B3 & 100 & 5.5 & 1.5 & 2 & 2 \\
\hline 4 & B4 & 100 & 6 & 0.5 & 2 & 2 \\
\hline 5 & B5 & 100 & 6 & 1.0 & 2 & 2 \\
\hline 6 & B6 & 100 & 6 & 1.5 & 2 & 2 \\
\hline 7 & B7 & 100 & 6.5 & 0.5 & 2 & 2 \\
\hline 8 & B8 & 100 & 6.5 & 1.0 & 2 & 2 \\
\hline 9 & B9 & 100 & 6.5 & 1.5 & 2 & 2 \\
\hline
\end{tabular}

\section{Preparation of Rifampicin loaded liquid crystals:}

For the preparation of Glycerol monooleate-based liquid crystals loaded with rifampicin, ingredients were taken according to above table. Rifampicin was firstly dissolved in $2 \mathrm{ml}$ of ethanol. Glycerol monooleate(GMO), P188 (P F-68), ethanol and water at a different ratios of (w/w). GMO was melted on a magnetig stirrer with heating plate with the temperature $60-80^{\circ} \mathrm{C}$. Then, the Rifampicin solution was added slowly on magnetic stirringat same temp. After complete addition of rifampicin solution stirred it continuously until total dissolution. Required amount of water was gradually added to melted mixture with magnetic stirrer for 20 minutes. The resulting viscous dispersions was sonicated/homogenized by using a probe sonicater/high-speed homogenizer (Ultra Turrax
T25) at certain high rpm to obtain andispersion of the LCs. Kept it for 24-48 hrs to form LC gel. ${ }^{\mathbf{1 1}}$

\section{Preparation of Granules of Rifampicin loaded LCs} as Final Dosage Form

The preparation of granules is carried out by wet granulation with sieving method by using sieve no 22 . Silicon dioxide (Aerosil) is used to form the dump mass. First the certain amount of SD was taken in mortar then LCs formulation is added slowly until the dump mass is obtained. Prepared dump mass is pass through the sieve and obtained granules are allow for drying at room temp.

\section{Characterization of Liquid crystals}

Particle Size Measurements: ${ }^{7-16}$ 
The particle size was determined by photon correlation spectroscopy using a Zetasizer Nano® (Malvern Instruments, Malvern, UK) at $25^{\circ} \mathrm{C}$. Samples were diluted with deionize water prior to the measurement. Particle size was analyzed by the dispersion technology software provided by Malvern instruments. The polydispersity index (PDI), which was dimensionless number indicating the width of the size distribution, was also obtained. ${ }^{7-16}$

\section{Zeta potential of the Rifampicin-LCs: ${ }^{17}$}

Zeta potential measurements were run at $25^{\circ} \mathrm{C}$ with electric field strength of $23 \mathrm{~V} / \mathrm{m}$, using Zetasizer (Nano ZS 90, Malvern Instruments, UK). To determine the zeta potential, samples of Rifampicin-LCs were diluted and placed in electrophoretic cell. The zeta potential was calculated as described by Helmholtz-Smoluchowski equation. ${ }^{17}$

\section{Scanning Electron Microscopy. ${ }^{18}$}

The surface morphology of liquid crystal nanoparticles was studied using a scanning electron microscopy (JSM 6390®, JEOL DATUM Ltd., Japan). Lyophilized powder of optimized formulations was dusted onto double-sided tape on an aluminum stub and coated with gold using a cold sputter coater in SEM chamber to a thickness of $400 \AA$, and then photomicrographs were captured by operating at an accelerating voltage of 15 $\mathrm{kV}$ electron beam. ${ }^{18}$

\section{X-Ray Diffraction ${ }^{9}$}

X-ray diffraction patterns of Rifampicin, Physical mixture and LCs containing Rifampicin(final dosage form) were obtained using X-ray Diffractometer (Brucker Axs, D8 Advance, and Germany) and $\mathrm{Cu}-\mathrm{K} \alpha$ line as a source of radiation which was operated at the voltage $40 \mathrm{kV}$ and the current $30 \mathrm{~mA}$. All samples were measured in the $2 \theta$ angle range between 100 and 600 with a scanning rate of $30 / \mathrm{min}$ and a step size of 0.020 .

\section{In vitro dissolution study}

The drug release study was carried out using a dissolution study apparatus (USP Apparatus I, basket type). The dissolution medium was having $\mathrm{pH} 1.2(0.1 \mathrm{~N}$ $\mathrm{HCl})$ and $\mathrm{pH} 6.8$ buffer. The dissolution experiments were conducted at $37 \pm 0.5^{\circ} \mathrm{C}$ at a basket rate of 100 rpm for 12 hours. Sampling was performed with interval of $0.5,1,2,3,4,5,6,7,8,9,10,11,12$ hours. first two hours in $\mathrm{pH} 1.2$ buffer $(0.1 \mathrm{~N} \mathrm{HCl})$ and next 3 to 12 hours in $\mathrm{pH} 6.8$ buffer .Detection of Rifampicin concentration in samples was identifying by using UVspectrophotometer at $331 \mathrm{~nm} .{ }^{19}$

\section{RESULT AND CONCLUSION}

\section{Drug-Excipient Interaction Study}

\section{Fourier transformed infrared spectroscopy}

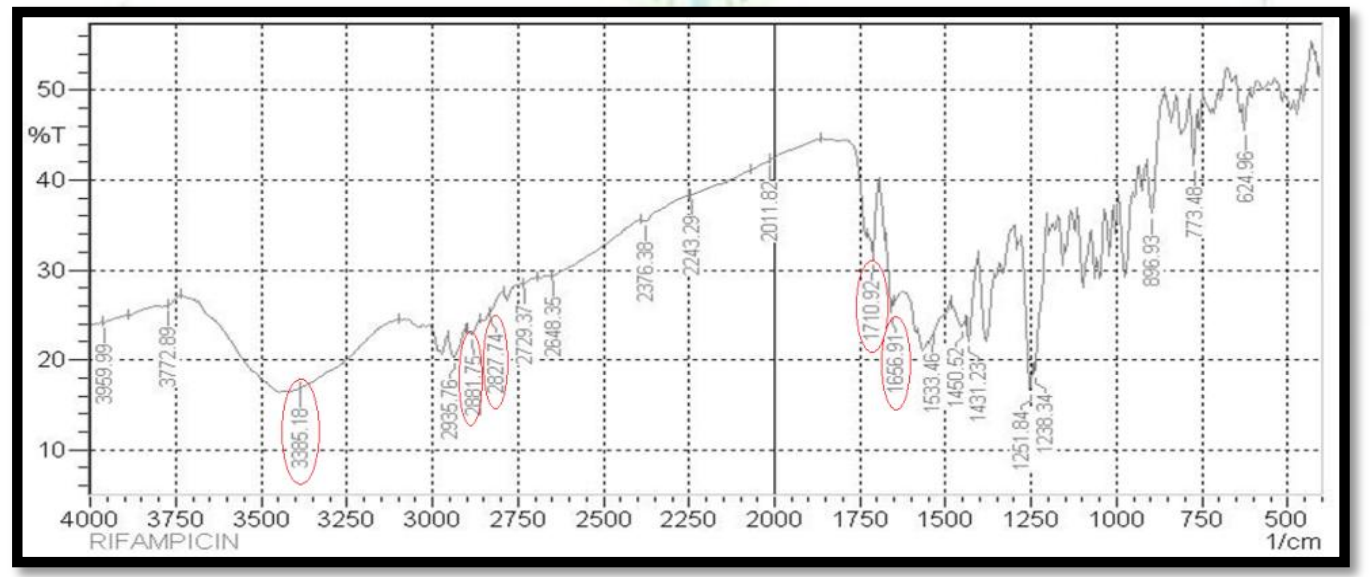

Figure 2: IR Spectra of Drug

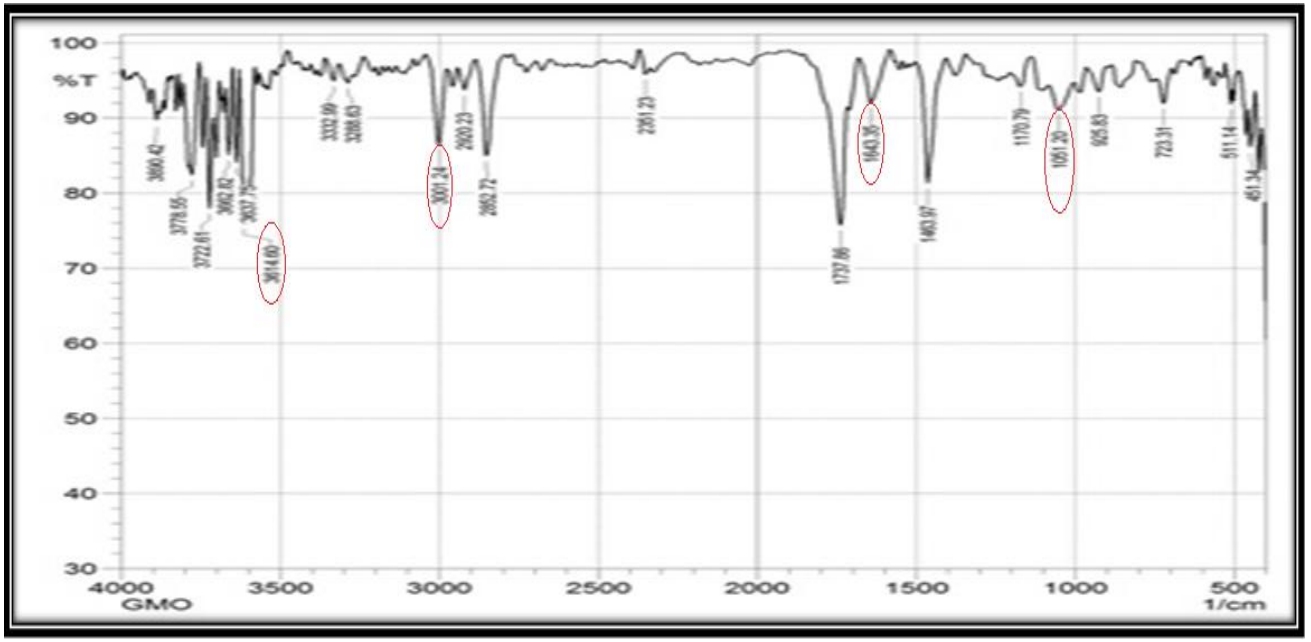

Figure 3: IR Spectra of GMO 


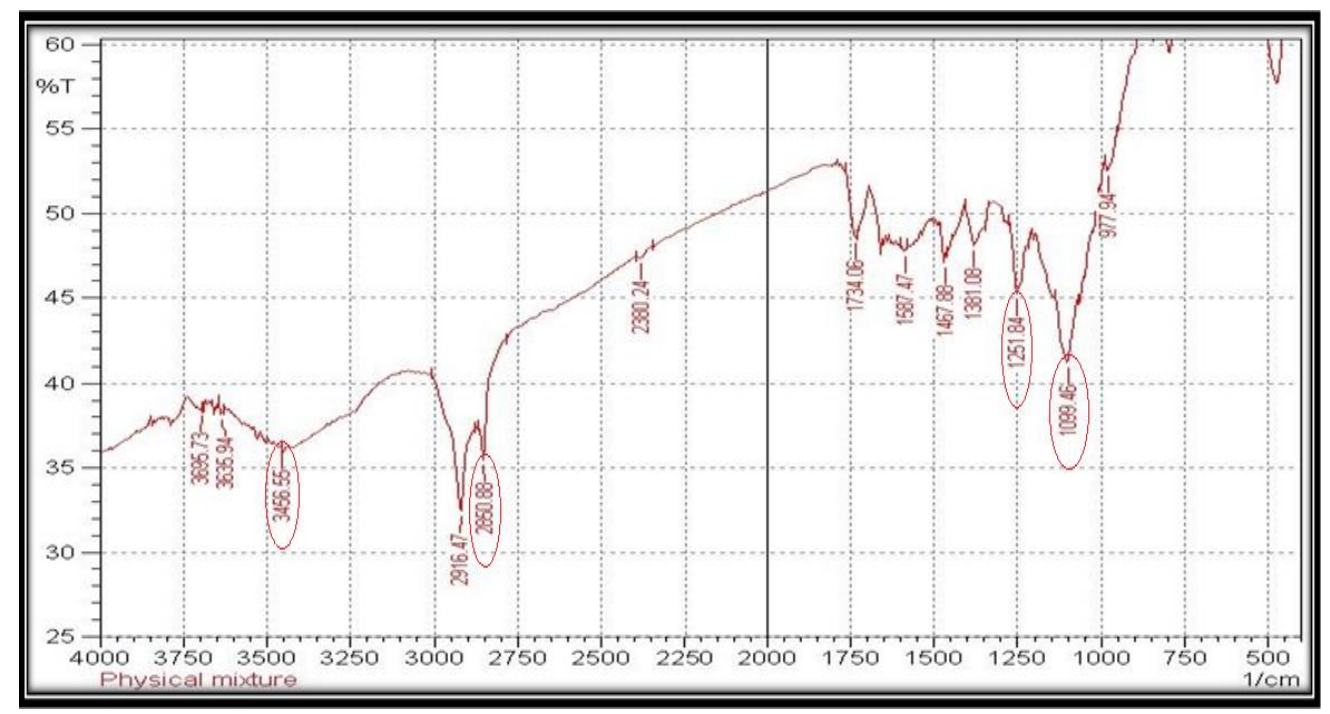

Figure 4: IR Spectra of Physical mixture

Table 2: Drug excipient interaction studies by IR spectroscopy

\begin{tabular}{|c|c|c|c|}
\hline \multirow{2}{*}{$\begin{array}{c}\text { Functional } \\
\text { Group }\end{array}$} & Drug & GMO & Physical mixture \\
\cline { 2 - 4 } & 3385.81 & 3637.75 & 3456.55 \\
\hline -OH Stretch & 2827.74 & 30001.24 & 2850.88 \\
\hline -CH Stretch & - & 1646.35 & - \\
\hline C=C Stretch & & 1051.20 & 1099.46 \\
\hline C-O Stretch & 2881.75 & - & 3456.55 \\
\hline N-H Stretch & 1656.91 & - & 1251.84 \\
\hline C-N Stretch & & & \\
\hline
\end{tabular}

There is no interaction was observed between drug and excipient by detection functional groups in physical mixture

\section{X -ray diffraction pattern (XRD)}

The Liquid crystals phase can be fragmented into stable submicron sized particles, which retain the internal structure of the original liquid crystals. As illustrated in Figure 3 and 4, pure cefixime trihydrate show fewer peaks as compare the Rifampicin-loaded liquid crystals showed X-ray diffraction (XRD) peaks in the X-ray diffraction curves, with the same spacing ratio.

\section{G RIFA (Coupled TwoTheta/Theta)}

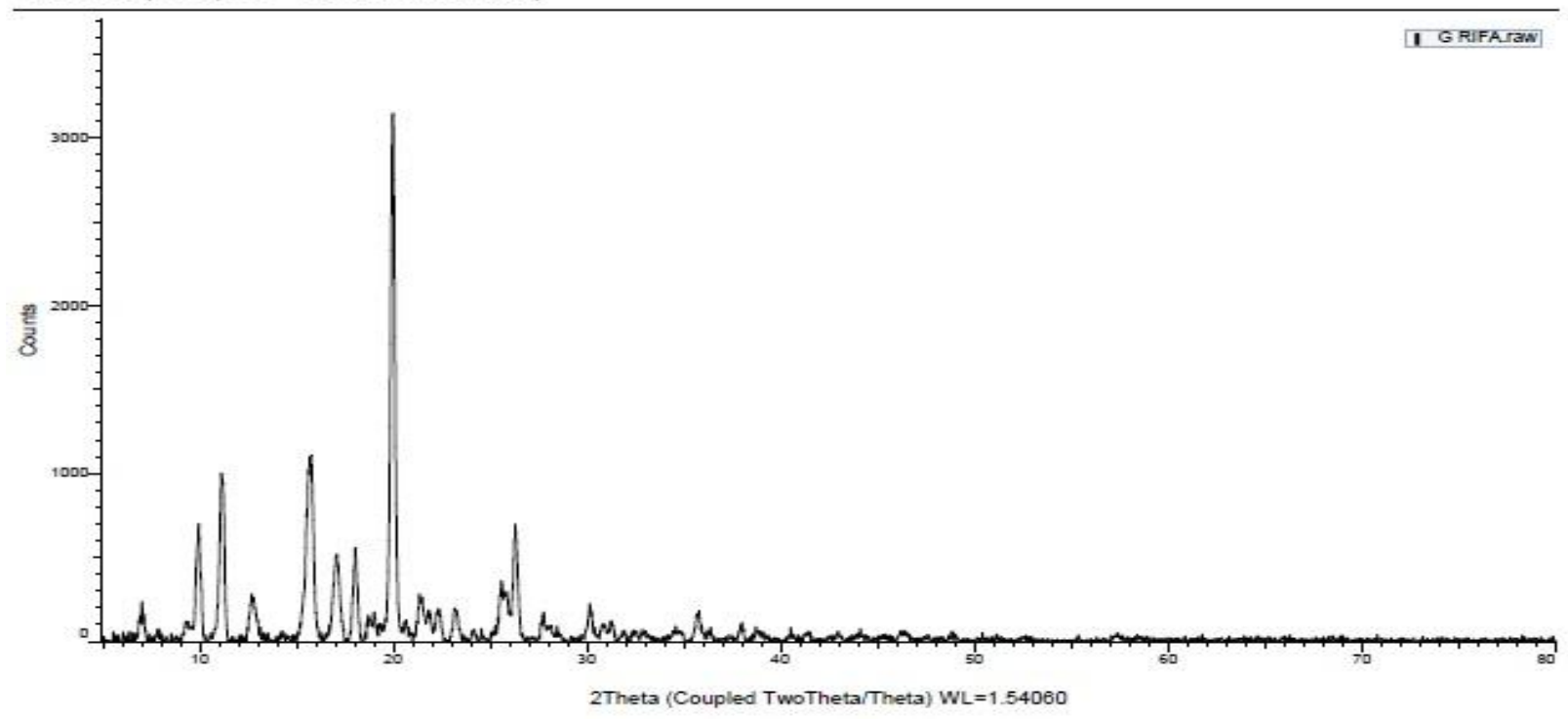

Figure 5: XRD of pure drug Rifampicin 


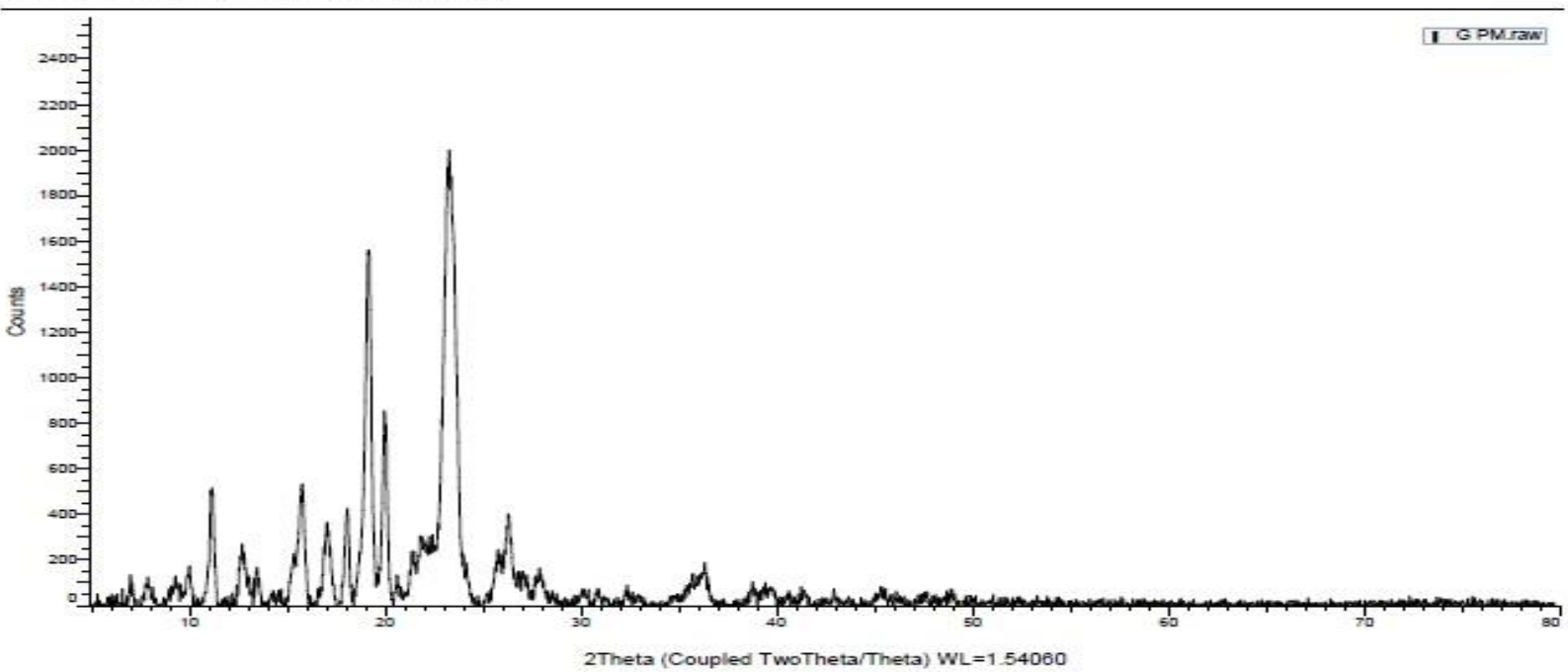

Figure 6: XRD of physical mixture

\section{G FORMULATION (Coupled TwoTheta/Theta)}

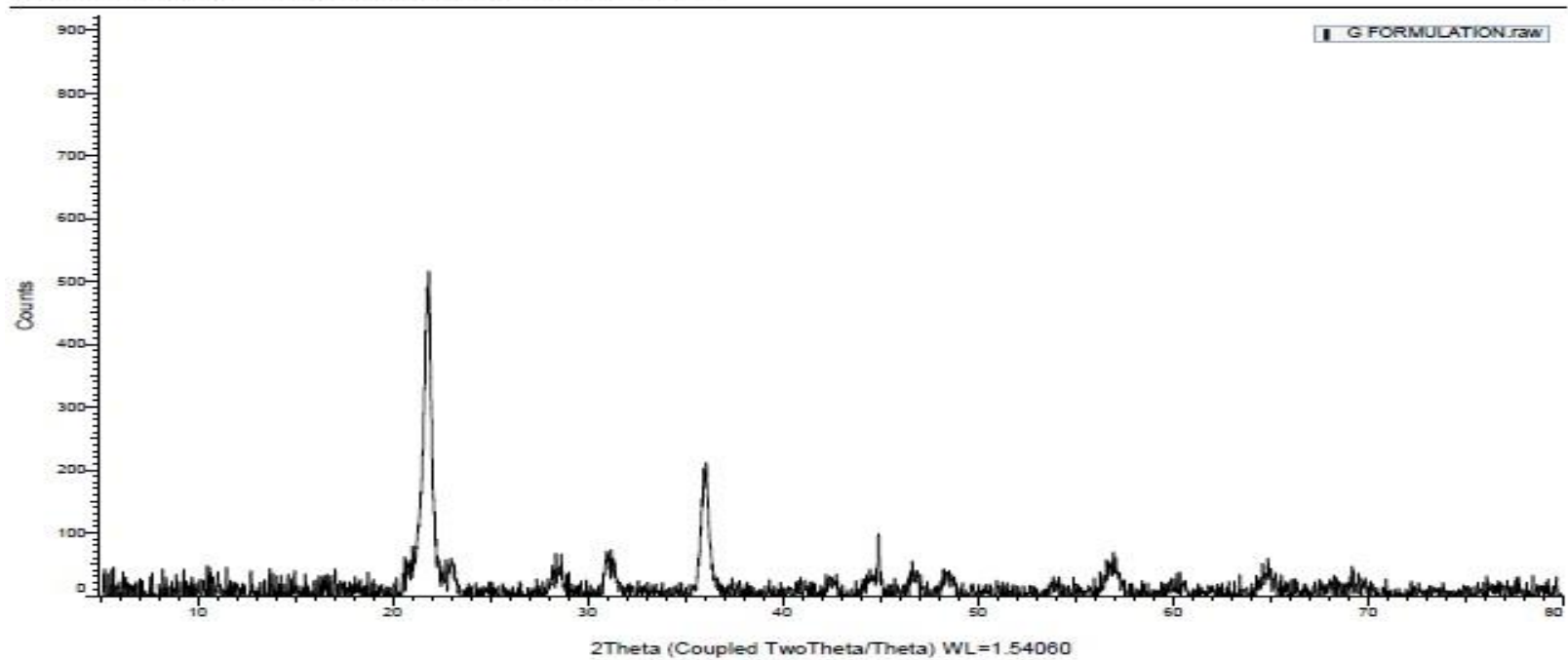

Figure 7: XRD of optimized formulation

\section{Scanning Electron Microscopy (SEM)}

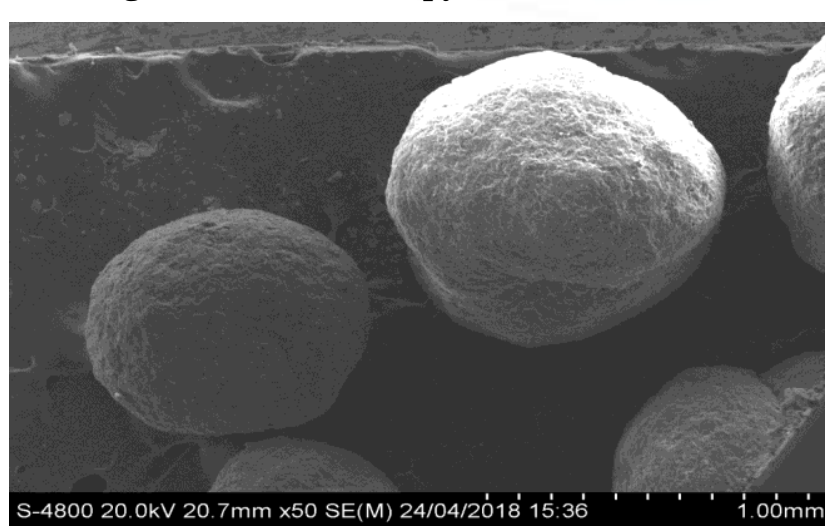

Figure 8: SEM image of liquid crystals granules loaded with rifampicin.

In order to further investigate the liquid crystals structure of the rifampicin GMO-based liquid crystals granules and liquid crystals containing rifampicin probe sonicated by ultra Tarrux T25 (B6), the formulations were examined by SEM.

Figure 8, showed the image of liquid crystal granules which contained reverse cubic structure with a mean particle size of about $168 \mathrm{~nm}$ close to that observed by laser diffraction. The image of liquid crystals containing Cefixime trihydrate with typical hexagonal characterization and a mean particle size of about 168 nm with PDI 0.367

\section{Zeta potential}

Zeta potential of optimized Rifampicin-LCs was found to be -40.1 as seen in Figure 5. It possesses negative surface charges due to the negatively charged CT. In addition to surfactant (Poloxamer 188) were also negatively charged. It is currently admitted that zeta potentials upto $-40.1 \mathrm{mV}$, are optimum and less than (60) $\mathrm{mV}$, are required for full electrostatic stabilization. 


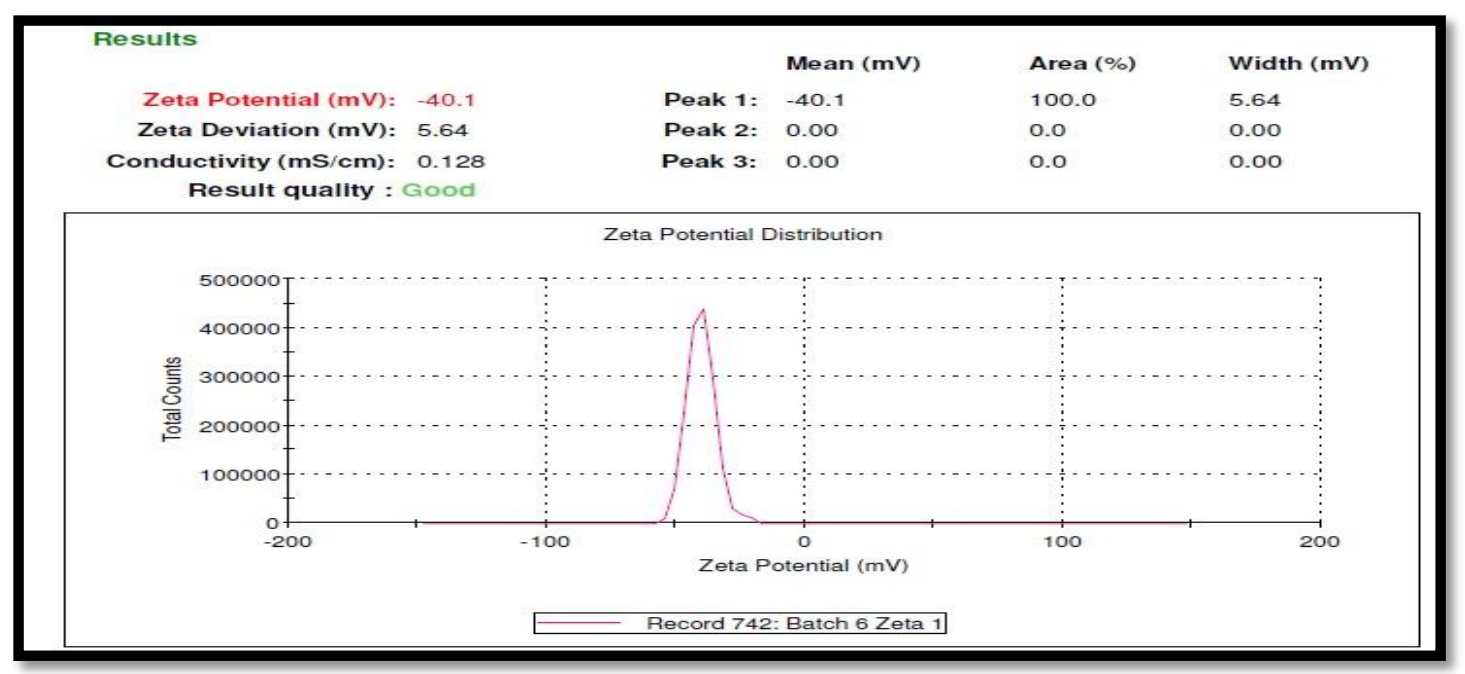

Figure 5: Zeta potential value of the optimized Rifampicin-LCs batch

\section{Mean Particle Size}

Particle size of optimized Rifampicin-LCs was found to be $168 \mathrm{~nm}$ as seen in Figure 6.

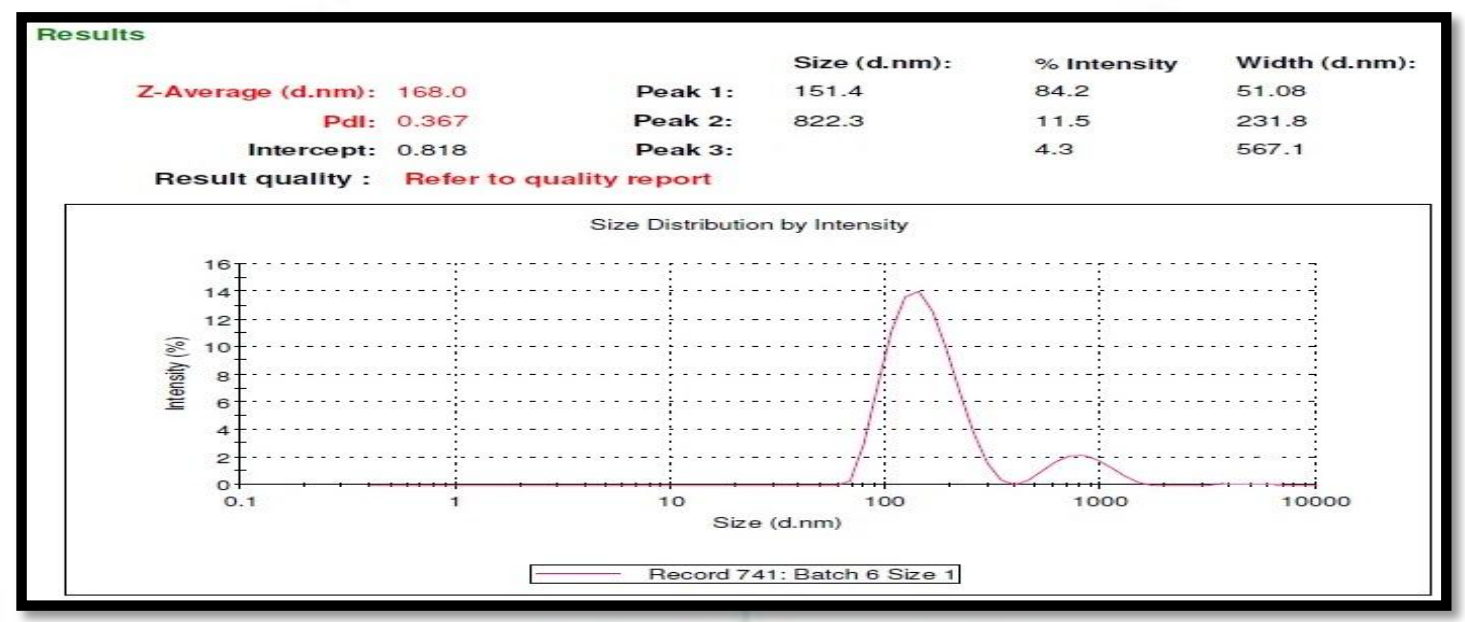

Figure 6: Particle size of the optimized Rifampicin-LCs batch

In-vitro dissolution Study of optimized formulation batch (B6):

In vitro drug release is determined for LCs loaded with Rifampicin in $1.2 \mathrm{pH}$ and $6.8 \mathrm{pH}$ phosphate buffer for 112 hours.

Table 3: In-vitro release data of optimized batch (B6)

\begin{tabular}{|c|c|}
\hline Time & \% CDR \\
\hline 0 & 0 \\
\hline 0.5 & 2.857143 \\
\hline 1 & 5.730159 \\
\hline 2 & 8.603175 \\
\hline 3 & 22.17857 \\
\hline 4 & 28.13459 \\
\hline 5 & 37.87302 \\
\hline 6 & 44.33135 \\
\hline 7 & 54.15774 \\
\hline 8 & 62.78737 \\
\hline 9 & 69.37996 \\
\hline 10 & 77.67394 \\
\hline 11 & 86.42857 \\
\hline 12 & 90.2295 \\
\hline
\end{tabular}

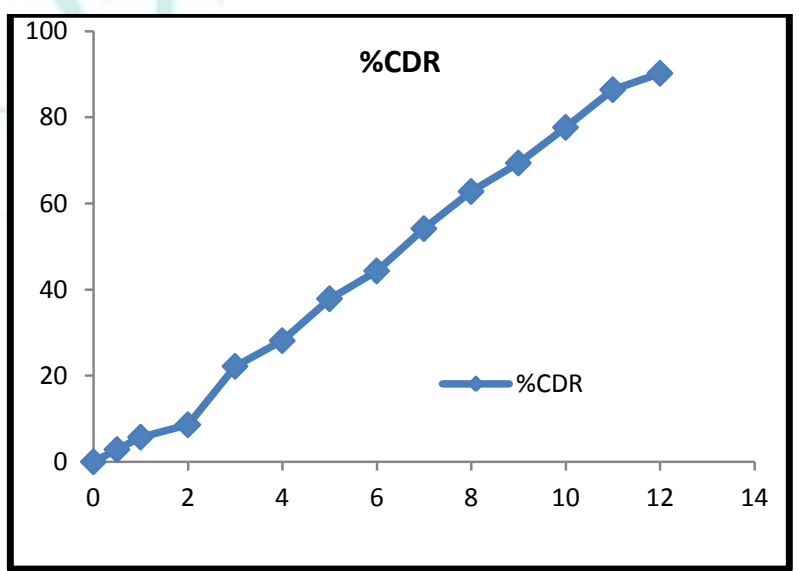

Figure 7: Graphical Representation of In-vitro release of optimized formulation batch (B6)

In-vitro Comparative dissolution study of optimized formulation batch with Rifampicin marketed formulation (Rifampicin 150):

For the determination of Comparison study study the marketed formulation of rifampicin $150 \mathrm{mg}$ capsule was selected and the in-vitro release was determined in 1.2 $\mathrm{pH}$ and $6.8 \mathrm{pH}$ buffer for 12 hours. In vitro drug release 
study of the optimized Rifampicin-LCs formulation. In the in vitro dissolution study show rifampicin loaded liquid crystal was improved the release rate and solubility as compared to marketed formulation.

Table 4: In-vitro release data of marketed formulation and optimize batch (B6)

\begin{tabular}{|c|c|c|}
\hline Time & $\begin{array}{c}\text { Marketed } \\
\text { Formulation }\end{array}$ & $\begin{array}{c}\text { Optimize } \\
\text { batch (B6) }\end{array}$ \\
\hline 0 & 0 & 0 \\
\hline 0.5 & 0 & 2.857 \\
\hline 1 & 4.285 & 5.730 \\
\hline 2 & 7.166 & 8.603 \\
\hline 3 & 18.813 & 22.178 \\
\hline 4 & 24.334 & 28.134 \\
\hline 5 & 30.301 & 37.873 \\
\hline 6 & 35.135 & 44.331 \\
\hline 7 & 42.366 & 54.157 \\
\hline 8 & 48.459 & 62.787 \\
\hline 9 & 52.667 & 69.379 \\
\hline 10 & 56.392 & 77.673 \\
\hline 11 & 60.396 & 86.428 \\
\hline 12 & 65.077 & 90.229 \\
\hline
\end{tabular}

\section{REFERENCES}

1. Kaufmann S.H. and McMichael, A.J., Annulling a dangerous liaison: vaccination strategies against AIDS and tuberculosis. Nature medicine, 2005; 11(4s):S33.

2. Sun Z., Zhang J., Song H., Zhang X., Li Y., Tian M., Liu Y., Zhao Y., Li C., Concomitant increases in spectrum and level of drug resistance in Mycobacterium tuberculosis isolates. International Journal of Tuberculosis Lung Disorders. 2010; 14:1436-1441.

3. Caminero J.A., Sotgiu G., Zumla A., Migliori G.B., Best drug treatment for multidrug-resistant and extensively drug-resistant tuberculosis. The Lancet Infectious Diseases. 2010; 10(9):621629.

4. Pooja D., Tunki L., Kulhari H., Reddy B.B. and Sistla R., 2015. Characterization, biorecognitive activity and stability of WGA grafted lipid nanostructures for the controlled delivery of Rifampicin. Chemistry and physics of lipids, 193, pp.11-17..

5. Muller F., Salonen A. and Glatter O. Monoglyceride-based cubosomes stabilized by laponite: Separating the effects of stabilizer, $\mathrm{pH}$ and temperature. Colloids Surf A: Physico Eng Asp, 2010; 358:50-56.

6. Gaikwad P.P. and Desai M.T. Liquid crystalline phase and its pharma applications. Intl J Pharma Res Rev, 2013; 2:40-52.

7. Lai J., Chen J., Lu Y., Sun J., Hu F., Yin Z. and Wu W. Glyceratemonooleate/poloxamer 407 cubic nanoparticles as oral drug delivery systems I. In vitro evaluation and enhanced oral bioavailability of the poorly water soluble drug Simvastatin. AAPS PharmSci Tech, 2009; 10:960-966.

8. Chen Y., Ma P and Gui S. Cubic and hexagonal liquid crystals as drug delivery systems. BioMed Research International, 2014; 1-12

9. Omray L.K. Liquid crystals as novel vesicular delivery system: Review. Curr Trends TechnolSci, 2013; 2:347-353.

10. Fong W.K, Dong, Y.D and Boyd, B.J., Drug delivery in Lyotropic Liquid Crystals: Applications of X-ray scattering in pharmaceutical science, Langmuir Int. J. Pharm. 2011.

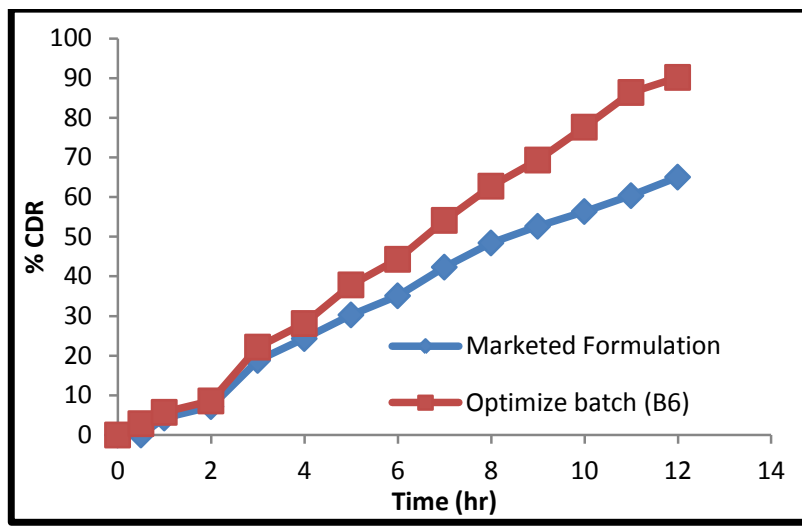

Figure 8: Graphical representation of in-vitro release of optimizes batch and marketed formulation.

\section{CONCLUSION}

Rifampicin was formulated as GMO based liquid crystals in an attempt to reverse cubic LCs having sustained release. An optimized formulation of GMO liquid crystals containing Rifampicin was characterized by the, particle size analysis, zeta potential and characterization was carried out by SEM and X-ray diffraction, following In-vitro dissolution and In-vitro. GMO-based cubosomes provided significant in the sustained drug delivery which helps to reduce frequency of dose.

11. Guo C., Wang J., Cao F., Lee R.J. and Zhai G. Lyotropic liquid crystal systems in drug delivery. Drug Del Today, 2010; 15:1032-1040.

12. Shah M.H. and Paradkar A. Cubic liquid crystalline glycerylmonooleate matrices for oral delivery of enzyme. Intl J Pharma 2005; 294:161-171.

13. Spicer P.T. Progress in liquid crystalline dispersions: Cubosomes. CurrOpin Colloid Inter Sci, 2005; 10:274-279.

14. Worle G., Siekmann B. and Bunjes H. Effect of drug loading on the transformation of vesicular into cubic nanoparticles during heat treatment of aqueous monoolein/poloxamer dispersions. Euro J PharmaBiopharma, 2006; 63:128-133.

15. Boyd B.J., Whittaker D.V., Khoo S.-M. and Davey G. Lyotropic liquid crystalline phases formed from glycerate surfactants as sustained release drug delivery systems. Intl $J$ Pharma 2006; 309:218-226.

16. Tilley A.J., Drummond C.J. and Boyd B.J. Disposition and association of the steric stabilizer Pluronic ${ }^{\circledR}$ F127 in lyotropic liquid crystalline nanostructured particle dispersions. J Colloid Inter Sci, 2013; 392:288-296.

17. Serpe, L., Catalano, M. G., Cavalli, R., Ugazio, E., Bosco, O., Canaparo, R., Muntoni, E., Frairia, R., Gasco, M. R., Eandi, M. and Zara, G. P. Cytotoxicity of anticancer drugs incorporated in solid lipid nanoparticles on HT-29 colorectal cancer cell line. Eur. J. Pharm. Biopharm., 2004; 58:673- 80.

18. Tan S. W., Billa N., Roberts C. R. and Burleyc J. C. Surfactant effects on the physical characteristics of Amphotericin Bcontaining nanostructured lipid carriers. Colloids Surf. A: Physicochem. Eng. Aspects. 2010; 372:73-9.

19. Siddique S., Khanam J. and Bigoniya P., Development of sustained release capsules containing "coated matrix granules of metoprolol tartrate". Aaps Pharmscitech, 2010; 11(3):13061314. 